\title{
GENERALIZED FEKETE MEANS
}

\author{
BY \\ PAUL SCHAEFER
}

1.0. Introduction. In 1954, M. Fekete [3] proposed two sequence-to-sequence summability methods which he called "Taylor-Nörlund" and "Nörlund-Taylor." His methods are extended in this paper by replacing the Taylor method with a more general summability method. Various inclusion properties of the new methods are developed. It is shown that these results hold when certain Hausdorff or quasi-Hausdorff methods are combined with Nörlund methods; in particular, for Cesàro, Euler or Taylor methods. Series-to-sequence analogues of these methods are defined and applied to the problem of summing the geometric series.

2.0. Preliminary definitions. An infinite matrix $\left(f_{n k}\right)$ is a $T$-matrix if and only if $t_{n}=\sum f_{n k} s_{k} \rightarrow s$ whenever $s_{n} \rightarrow s$. An infinite matrix $\left(a_{n k}\right)$ is an alphamatrix if and only if $\sum v_{n}=s$, where $v_{n}=\sum a_{n k} u_{k}$, whenever $\sum u_{n}=s$. The infinite matrix $\left(g_{n k}\right)$ is a gamma-matrix if and only if $t_{n}=\Sigma g_{n k} u_{k} \rightarrow s$ whenever $\Sigma u_{n}=s$. Given a $T$-matrix $\left(f_{n k}\right)$, it has been shown [13], [1, p. 86] that if $g_{n k}=\sum_{j=k}^{\infty} f_{n j}$, if $a_{0 k}=g_{0 k}$ and if $a_{n k}=g_{n k}-g_{n-1, k}$ when $n \geqq 1$, then $\left(g_{n k}\right)$ is a gamma-matrix and $\left(a_{n k}\right)$ is an alpha-matrix such that $\lim _{n} \sum_{k} g_{n k} u_{k}=\sum_{n} \sum_{k} a_{n k} u_{k}$ $=s$ whenever $\lim _{n} \sum_{k} f_{n k} s_{k}=s$ for bounded $s_{n}=u_{0}+\cdots+u_{n}$, at least. Such triples of $T$-, gamma- and alpha-matrices define a regular summability method $A$. When $\lim _{n} \sum_{k} f_{n k} s_{k}=s$, it is convenient to write $A$-lim $s_{n}=s$.

A particular class of regular methods, the Nörlund methods, is of importance to the subsequent discussion. A sequence $\left\{p_{n}\right\}$ of real numbers is a Nörlund sequence [3] if and only if $p_{0}>0, p_{n} \geqq 0$ for $n \geqq 1$, and $p_{n} / P_{n} \rightarrow 0$, where $P_{n}=p_{0}+\cdots+p_{n}$. Given a Nörlund sequence $\left\{p_{n}\right\}$, let $F_{n k}=p_{n-k} / P_{n}$ if $n \geqq k, 0$ otherwise. Then $\left(F_{n k}\right)$ is a $T$-matrix $[4, \mathrm{p} .64]$ and the summability method defined by it is called the regular Nörlund method $\left(N, p_{n}\right)$.

The summability method $A$ is said to include the summability method $B$ if $A$-lim $s_{n}=B$-lim $s_{n}$ for every sequence $\left\{s_{n}\right\}$ having a convergent $B$-limit. $A$ totally includes $B$ if $B-\lim s_{n}=s$ implies that $A-\lim s_{n}=s$ for every $s$, finite or infinite [12].

\subsection{The $A$-Nörlund method.}

\subsection{THE DEFINITION.}

Definition 3.1.1. Let $A$ be a regular summability method with alpha-matrix $\left(a_{n k}\right)$ and $T$-matrix $\left(f_{n k}\right)$. Let $\left(N, p_{n}\right)$ be a regular Nörlund method such that $\left(N, q_{n}\right)$ is a regular Nörlund method, where $q_{n}=\sum a_{n k} p_{k}$. A sequence $\left\{s_{n}\right\}$ is said

Received by the editors April 1, 1964 and, in revised form, February 3, 1965. 
to be summable to $s$ by the $A$-Nörlund method if and only if $t_{n} \rightarrow s,\left(N, q_{n}\right)$, where $t_{n}=\sum f_{n k} s_{k}$. Write $s_{n} \rightarrow s, F\left(A, p_{n}\right)$ in this case.

It may happen that the transform of a Nörlund sequence by an alpha-matrix is not a Nörlund sequence. For example, let $\left(a_{n k}\right)$ be an alpha-matrix with $a_{u v}<0$ for some positive integers $u$ and $v$. Define a Nörlund sequence $\left\{p_{n}\right\}$, where $p_{0}=1, p_{v}>\left|a_{u 0} / a_{u v}\right|, p_{n}=0$ otherwise. Then $q_{u}=a_{u 0}+a_{u v} p_{v}<0$ and $\left\{q_{n}\right\}$ clearly is not a Nörlund sequence. The next two lemmas provide conditions that $\left\{q_{n}\right\}$ be a Nörlund sequence.

LemmA 3.1.2. If the alpha-matrix $\left(a_{n k}\right)$ of $A$ satisfies $a_{00}>0, a_{n k} \geqq 0$ otherwise; if there exists a constant $M>0$ such that $a_{n k} \leqq M \cdot f_{n k}$, where $\left(f_{n k}\right)$ is the $T$-matrix of $A$; if $\left\{p_{n}\right\}$ is a Nörlund sequence such that $q_{n}=\sum a_{n k} p_{k}$ exists for every $n$, then $\left\{q_{n}\right\}$ is a Nörlund sequence.

Proof. Clearly, $q_{n} \geqq 0$ and $q_{0} \geqq a_{00} p_{0}>0$. If $q_{0}+\cdots+q_{n}=Q_{n}$, then

$$
\begin{aligned}
Q_{n} & =\sum_{k=0}^{n} \sum_{j=0}^{\infty} a_{k j} p_{j}=\sum_{j=0}^{\infty} \sum_{k=0}^{n} a_{k j} p_{j} \\
& =\sum_{j=0}^{\infty} g_{n j} p_{j}=\sum_{j=0}^{\infty} \sum_{k=j}^{\infty} f_{n k} p_{j}=\sum_{k=0}^{\infty} f_{n k} \cdot \sum_{j=0}^{k} p_{j}=\sum_{k=0}^{\infty} f_{n k} P_{k},
\end{aligned}
$$

where $\left(g_{n k}\right)$ is the gamma-matrix of $A$, the inversions of order of summation being justified, since all terms are non-negative. Then, $q_{n} / Q_{n}=\left(\sum a_{n k} p_{k}\right) /\left(\sum f_{n k} P_{k}\right)$ $=\sum h_{n k}\left(p_{k} / P_{k}\right)$, where $h_{n k}=\left(a_{n k} P_{k}\right) /\left(\sum f_{n k} P_{k}\right)$. Now, $\sum_{k}\left|h_{n k}\right|=\sum_{k} h_{n k} \leqq$ $\left(M \cdot \sum f_{n k} P_{k}\right) /\left(\sum f_{n k} P_{k}\right)=M$ for every $n$. Also, $h_{n k}=\left(a_{n k} P_{k}\right) / Q_{n} \leqq\left(M \cdot f_{n k} P_{k}\right) / q_{0}$ $\rightarrow 0$ as $n \rightarrow \infty$ for every $k$. Therefore, by Theorem 4 of [4], $q_{n} / Q_{n} \rightarrow 0$ if $p_{n} / P_{n} \rightarrow 0$. Hence, $\left\{q_{n}\right\}$ is a Nörlund sequence.

A slight modification of the discussion preceding the lemma shows that $a_{00}>0$ and $a_{n k} \geqq 0$ otherwise are necessary for $\left\{q_{n}\right\}$ to be a Nörlund sequence. An example is given after Lemma 3.4.1 below to show that the second condition on the matrices $\left(a_{n k}\right)$ and $\left(f_{n k}\right)$ is not a necessary condition.

LEMMA 3.1.3. Let $\left(a_{n k}\right)$ be an alpha-matrix and let $\left\{p_{n}\right\}$ be a Nörlund sequence such that $\sum p_{n}$ converges and $q_{n}=\sum a_{n k} p_{k}$ exists for every $n$. Then, the conditions $a_{00}>0$ and $a_{n k} \geqq 0$ otherwise are necessary and sufficient that $\left\{q_{n}\right\}$ be a Nörlund sequence.

Proof. The necessity of the conditions is obvious. For sufficiency, one notes that $\sum q_{n}$ converges and hence that $q_{n} / Q_{n} \leqq q_{n} / q_{0} \rightarrow 0$.

In particular, Lemma 3.1.3 shows that finite Nörlund sequences, when $p_{n} \neq 0$ for only finitely many $n$, are transformed into Nörlund sequences by any alphamatrix $\left(a_{n k}\right)$ with non-negative entries and $a_{00}>0$.

3.2. Properties of $F\left(A, p_{n}\right)$. Since the Nörlund method $\left(N, q_{n}\right)$ is regular, if $t_{n} \rightarrow s$, then $t_{n} \rightarrow s,\left(N, q_{n}\right)$. Thus the next theorem is an immediate consequence of the definition of $F\left(A, p_{n}\right)$. 
THEOREM 3.2.1. $F\left(A, p_{n}\right)$ is regular and includes $A$.

In fact, since the matrix of $\left(N, q_{n}\right)$ has non-negative elements, if $t_{n}$ exists for every $n$ and $t_{n} \rightarrow+\infty$, it follows that $t_{n} \rightarrow+\infty,\left(N, q_{n}\right)[4$, Theorem 9$]$, so $F\left(A, p_{n}\right)$ totally includes $A$.

If $A$ is $(C, 1)$, if $p_{0}=1, p_{n}=2$ for $n \geqq 1$, and if $s_{n}=(n+1)(-1)^{n}$ then $\left\{s_{n}\right\}$ is not summable $A$, but $s_{n} \rightarrow 0, F\left(A, p_{n}\right)$, as a short calculation shows. Hence, in general, $F\left(A, p_{n}\right)$ is not equivalent to the method $A$.

Since Nörlund methods are consistent $[4$, p. 65], it follows that if $s_{n} \rightarrow s, F\left(A, p_{n}\right)$ and $s_{n} \rightarrow s^{\prime}, F\left(A, r_{n}\right)$, then $s=s^{\prime}$. Thus, for fixed $A$ and various Nörlund sequences, the methods $F\left(A, p_{n}\right)$ are consistent.

However, if $A$ and $B$ are inconsistent methods, if $s_{n} \rightarrow s, F\left(A, p_{n}\right)$ and $s_{n} \rightarrow s^{\prime}$, $F\left(B, p_{n}\right)$, it need not follow that $s=s^{\prime}$ when $\left\{s_{n}\right\}$ is divergent. For example, let $A$ transform $\left\{s_{n}\right\}$ into the sequence $\left\{t_{n}\right\}$, where $t_{n}=\left(s_{2 n}+s_{2 n+2}\right) / 2$, and let $B$ transform $\left\{s_{n}\right\}$ into the sequence $\left\{u_{n}\right\}$, where $u_{n}=\left(s_{2 n+1}+s_{2 n+3}\right) / 2$. Then, a short calculation shows that the alpha-matrices of $A$ and $B$ satisfy the hypotheses of Lemma 3.1.3. If $\left\{p_{n}\right\}$ is any finite Nörlund sequence, it follows from Theorem 3.2.1 that $(-1)^{n} \rightarrow 1, F\left(A, p_{n}\right)$ while $(-1)^{n} \rightarrow-1, F\left(B, p_{n}\right)$.

Definition 3.2.2. [14, p. 111]. A sequence $\left\{s_{n}\right\}$ is summable to $s$ by the strongAbel method if and only if $s^{*}(x)=(1-x) \cdot \sum s_{n} x^{n}$ has a positive radius of convergence and defines an analytic function which is regular for $0 \leqq x<1$ and which tends to $s$ as $x \rightarrow 1$ through real values less than 1 .

THEOREM 3.2.3. If $s_{n} \rightarrow s, F\left(A, p_{n}\right)$, then $s_{n} \rightarrow s$ by the product of $A$ and the strong-Abel methods.

Proof. Let $v_{0}=t_{0}, v_{n}=t_{n}-t_{n-1}$ for $n \geqq 1$. Then, if $s_{n} \rightarrow s, F\left(A, p_{n}\right), \sum v_{n}=s$, $\left(N, q_{n}\right)$. By Theorem 18 of [4], $\Sigma v_{n}=s$ by the strong-Abel method. Since $t^{*}(x)=v(x)=\sum v_{n} x^{n}$, the assertion follows.

If it should happen that $s_{n} \rightarrow s, F\left(A, p_{n}\right)$ and $\sum t_{n} x^{n}$ converges for $|x|<1$, then $s_{n} \rightarrow s$ by the product of $A$ and the Abel method. That $F\left(A, p_{n}\right)$ is not, in general, equivalent to the product of $A$ and the (strong-)Abel methods can be seen by letting $A$ be $(C, 1), p_{0}=1, p_{1}=2, p_{2}=1, p_{n}=0$ for $n \geqq 3$, and $s_{n}=(n+1)(-1)^{n}$. It can be seen, after some calculations, that $s_{n} \rightarrow 0$ by the product of the $(C, 1)$ and Abel methods, but $\left\{s_{n}\right\}$ is not summable $F\left(A, p_{n}\right)$.

DeFINITION 3.2.4. An alpha-matrix $\left(a_{n k}\right)$ is said to be monotone-preserving with respect to the nondecreasing sequence $\left\{p_{n}\right\}$ if and only if the sequence $\left\{q_{n}\right\}=\left\{\sum a_{n k} p_{k}\right\}$ is nondecreasing.

Definition 3.2.5. An alpha-matrix $\left(a_{n k}\right)$ is said to be $(C, 1)$-preserving if and only if $\left(N, q_{n}\right)$ is equivalent to $(C, 1)$ whenever $\left(N, p_{n}\right)$ is $(C, 1)$ and $q_{n}=\sum a_{n k} p_{k}=\sum a_{n k}$.

Clearly, if $\sum_{k} a_{n k}=L>0$ for every $n$, then $\left(a_{n k}\right)$ is $(C, 1)$-preserving, since $q_{0}=q_{1}=\cdots=L$. 
THEOREM 3.2.6. If the alpha-matrix of $A$ is $(C, 1)$-preserving and monotonepreserving with respect to the nondecreasing Nörlund sequence $\left\{p_{n}\right\}$, then $F\left(A, p_{n}\right)$ includes $F(A, 1)$.

Proof. Since $\left\{q_{n}\right\}$ is nondecreasing, $\left(N, q_{n}\right)$ includes $(C, 1)$, by Theorem 20 of [4]. If $s_{n} \rightarrow s, F(A, 1)$, then $t_{n} \rightarrow s,\left(N, r_{n}\right)$, where $\left(N, r_{n}\right)$ is equivalent to $(C, 1)$. Thus, $t_{n} \rightarrow s,(C, 1)$ and therefore $t_{n} \rightarrow s,\left(N, q_{n}\right)$.

THEOREM 3.2.7. If the method $A$ is such that the A-transform of a sequence $\left\{s_{n}\right\}$ is summable to $s$ by the Abel method whenever $\left\{s_{n}\right\}$ is summable to s by the Abel method, if the alpha-matrix of $A$ is monotone-preserving with respect to the nondecreasing Nörlund sequence $\left\{p_{n}\right\}$, then $F\left(A, p_{n}\right)$ includes $(C, 1)$ for bounded sequences.

Proof. Let $s_{n} \rightarrow s,(C, 1)$. Then $s_{n} \rightarrow s,(\mathrm{Abel})$, so $t_{n} \rightarrow s,(\mathrm{Abel})$. Since $\left\{s_{n}\right\}$ is bounded, $\left\{t_{n}\right\}$ is bounded. Hence, by Theorem 92 of $[4], t_{n} \rightarrow s,(C, 1)$. Since $\left\{q_{n}\right\}$ is a nondecreasing Nörlund sequence, $\left(N, q_{n}\right)$ includes $(C, 1)$, and therefore, $t_{n} \rightarrow s,\left(N, q_{n}\right)$.

The methods $\left(N, p_{n}\right)$ and $F\left(A, p_{n}\right)$, in general, are not comparable. It has already been noted that when $A$ is $(C, 1)$, and $p_{0}=1, p_{1}=2, p_{2}=1, p_{n}=0$ for $n \geqq 3$, the sequence $\left\{(n+1)(-1)^{n}\right\}$ is not summable $F\left(A, p_{n}\right)$, although $(n+1)(-1)^{n}$ $\rightarrow 0,\left(N, p_{n}\right)$. On the other hand, for the same $A$ and $\left\{p_{n}\right\}$, if $r_{n}=2 i^{n}$, then $\left\{r_{n}\right\}$ is not summable $\left(N, p_{n}\right)$, although $r_{n} \rightarrow 0,(C, 1)=A$, and hence $r_{n} \rightarrow 0, F\left(A, p_{n}\right)$.

3.3. HAUSDORFF-NÖLUND METHODS. Let $\left\{\mu_{n}\right\}$ be a sequence of real numbers such that $\mu_{0}=1, \Delta^{n} \mu_{0} \rightarrow 0$ and $\Delta^{p} \mu_{n} \geqq 0$ for all $n$ and $p$, where $\Delta \mu_{n}=\mu_{n}-\mu_{n+1}$, $\Delta^{p+1} \mu_{n}=\Delta\left(\Delta^{p} \mu_{n}\right)$. The matrix $\left(f_{n k}\right)$, where $f_{n k}=\left(\begin{array}{l}n \\ k\end{array}\right) \Delta^{n-k} \mu_{k}$ if $n \geqq k, 0$ otherwise, is a $T$-matrix $[14$, p. 148$]$. Associated with this $T$-matrix is the alpha-matrix $\left(a_{n k}\right)$, where $a_{0 k}=f_{0 k}, a_{n k}=(k / n) f_{n k}$ when $n \geqq 1$. Let $H\left(\mu_{n}\right)$ denote the regular summability method determined by these matrices.

The $H\left(\mu_{n}\right)$ methods are a subclass of the set of regular Hausdorff methods. This subclass includes the Cesàro, Hölder, and Euler methods [4, Chapter XI], and hence is of some interest. Since the $T$-matrix for $H\left(\mu_{n}\right)$ has non-negative elements, it is a totally regular matrix [4, Theorem 9] and thus, $\mu_{n} \neq 0$ for every $n$ [12]. The alpha-matrix of $H\left(\mu_{n}\right)$ transforms Nörlund sequences into Nörlund sequences, for $q_{n}$ always exists and Lemma 3.1.2 holds with $M=1$. One can therefore define $H\left(\mu_{n}\right)$-Nörlund methods for any Nörlund sequence. In order to see how the results of $\$ 3.2$ hold for these Hausdorff-Nörlund methods, one observes that Pati [8] has shown that if $\left\{s_{n}\right\}$ is summable by the Abel method, the $H\left(\mu_{n}\right)-$ transform of $\left\{s_{n}\right\}$ is also summable by the Abel method to the same sum. Regarding the $(C, 1)$ - and monotone-preserving properties, there are the following results.

LEMMA 3.3.1. The alpha-matrix of $H\left(\mu_{n}\right)$ is $(C, 1)$-preserving. 
Proof. $\quad \sum_{k} a_{0 k}=1, \quad \sum_{k} a_{n k}=\sum_{k=1}^{n}\left(\begin{array}{c}n-1 \\ k-1\end{array}\right) \Delta^{n-k} \mu_{k}=\mu_{1} \quad$ for $\quad n \geqq 1, \quad$ and $0<\mu_{1} \leqq 1$. If $p_{n}=1$ for all $n$, so $\left(N, p_{n}\right)$ is $(C, 1)$, then $q_{0}=1$ and $q_{n}=\mu_{1}$ for $n \geqq 1$. Let $q(x)=\sum q_{n} x^{n}=1+\left(\mu_{1} x\right) /(1-x)$ and $p(x)=\sum p_{n} x^{n}=1 /(1-x)$, so $q(x) / p(x)=1-\left(1-\mu_{1}\right) x=\sum K_{n} x^{n}$, and $p(x) / q(x)=1 /\left[1-\left(1-\mu_{1}\right) x\right]=$ $\sum\left(1-\mu_{1}\right)^{n} x^{n}=\Sigma L_{n} x^{n}$. Since both $\Sigma\left|K_{n}\right|$ and $\Sigma\left|L_{n}\right|$ converge, $\left(N, q_{n}\right)$ is equivalent to $\left(N, p_{n}\right)=(C, 1)$ by Theorem 21 of [4].

LEMMA 3.3.2. The alpha-matrix of $H\left(\mu_{n}\right)$ is monotone-preserving with respect to those nondecreasing sequences $\left\{p_{n}\right\}$ for which $p_{0} \leqq \mu_{1} p_{1}$.

Proof. $q_{0}-q_{1}=p_{0}-\mu_{1} p_{1} \leqq 0$. For $n \geqq 1$,

$$
q_{n}-q_{n+1}=\sum_{k=1}^{n}\left(\begin{array}{c}
n-1 \\
k-1
\end{array}\right) \Delta^{n-k} \mu_{k} p_{k}-\sum_{k=1}^{n+1}\left(\begin{array}{c}
n \\
k-1
\end{array}\right) \Delta^{n-k+1} \mu_{k} p_{k},
$$

which, after some simplifications, becomes

$$
q_{n}-q_{n+1}=\sum_{k=1}^{n}\left(\begin{array}{l}
n-1 \\
k-1
\end{array}\right) \Delta^{n-k} \mu_{k+1}\left(p_{k}-p_{k+1}\right) .
$$

Since $\Delta^{p} \mu_{n} \geqq 0$, it follows that $q_{n}-q_{n+1} \leqq 0$ if $\left\{p_{n}\right\}$ is nondecreasing. Thus, the sequence $\left\{q_{n}\right\}$ is nondecreasing.

3.4. QUASI-HAUSDORFF-NÖRLUND METHODS. Let $G(u)$ be a nondecreasing function on $[0,1]$ such that $G(0)=G(0+)=0, G(1)=1$ and $\int_{0}^{1}(1 / u) d G$ exists. Let $\mu_{n}=\int_{0}^{1} u^{n} d G$. The matrix $\left(f_{n k}\right)$, where $f_{n k}=\left({ }_{n}^{k}\right) \Delta^{k-n} \mu_{n+1}$ if $k \geqq n, 0$ otherwise, is a $T$-matrix [9], [10]. Associated with this $T$-matrix is the alpha-matrix $\left(a_{n k}\right)$, where $a_{n k}=\left(\begin{array}{l}k \\ n\end{array}\right) \Delta^{k-n} \mu_{n}$ if $k \geqq n, 0$ otherwise. Let $H^{*}\left(\mu_{n}\right)$ denote the regular summability method determined by these matrices.

These $H^{*}\left(\mu_{n}\right)$ methods are a subclass of the set of regular quasi-Hausdorff methods. This subclass includes the Taylor method. Since the alpha-matrix of $H^{*}\left(\mu_{n}\right)$ method has $a_{00}=1$ and $a_{n k} \geqq 0$ and has bounded row-sums, it follows from Lemma 3.1.3 that $H^{*}\left(\mu_{n}\right)$-Nörlund methods may be defined for any Nörlund sequence for which $\Sigma p_{n}$ converges. The next lemma deals with general Nörlund sequences.

Lemma 3.4.1. If there exists $a b>0$ such that $G(u)=0$ for $0 \leqq u \leqq b$, then the alpha-matrix of $H^{*}\left(\mu_{n}\right)$ transforms Nörlund sequences into Nörlund sequences.

Proof. Let $p(x)=\Sigma p_{n} x^{n}$, where $\left\{p_{n}\right\}$ is a Nörlund sequence. This series converges for $|x|<1[4$, p. 65]. Hence, for every $n$,

$$
\int_{0}^{1}\left(\frac{u^{n}}{n !}\right) \cdot p^{(n)}(1-u) d G=\sum_{k=n}^{\infty}\left(\begin{array}{c}
k \\
n
\end{array}\right) p_{k} \cdot \int_{0}^{1} u^{n}(1-u)^{k-n} d G=q_{n}
$$

exists, the interchange of summation and integration being justified, since the integrands are non-negative and continuous on $[b, 1]$. Furthermore, for $k \geqq n$, 


$$
\begin{aligned}
f_{n k}-b \cdot a_{n k} & =\left(\begin{array}{l}
k \\
n
\end{array}\right) \int_{0}^{1} u^{n+1}(1-u)^{k-n} d G-\left(\begin{array}{l}
k \\
n
\end{array}\right) \int_{0}^{1} b \cdot u^{n}(1-u)^{k-n} d G \\
& =\left(\begin{array}{l}
k \\
n
\end{array}\right) \int_{b}^{1} u^{n}(1-u)^{k-n}(u-b) d G \geqq 0 .
\end{aligned}
$$

Thus, $a_{n k} \leqq(1 / b) \cdot f_{n k}$ for all $n$ and $k$, and the hypotheses of Lemma 3.1.2 are satisfied.

In order to see that the hypothesis of Lemma 3.4.1 is not a necessary condition, let $H^{*}\left(\mu_{n}\right)$ be generated by $G(u)=u^{2}, 0 \leqq u \leqq 1$. Then $\mu_{n}=2 /(n+2)$. A short calculation shows that the alpha-matrix $\left(a_{n k}\right)$ of $H^{*}\left(\mu_{n}\right)$ is given by $a_{n k}=2(n+1) /(k+1)(k+2)$ for $k \geqq n, 0$ otherwise; and the $T$-matrix $\left(f_{n k}\right)$ of this method is given by $f_{n k}=2(n+1)(n+2) /(k+1)(k+2)(k+3)$ for $k \geqq n$, 0 otherwise. If $\left\{p_{n}\right\}$ is any sequence of non-negative terms with $p_{0}>0$ such that $q_{n}=\sum a_{n k} p_{k}$ exists for every $n$, the proof of Lemma 3.1.2 shows that $Q_{n}=q_{0}+\cdots+q_{n}=\sum f_{n k} P_{k}$, where $P_{n}=p_{0}+\cdots+p_{n}$. It is easily seen for this method that $0 \leqq q_{n}=Q_{n}-Q_{n-1}=2 Q_{n} /(n+2)-2 P_{n-1} /(n+2)<2 Q_{n} /(n+2)$, so $q_{n} / Q_{n} \rightarrow 0$ and $\left\{q_{n}\right\}$ is a Nörlund sequence. Furthermore, since $(n+2) a_{n k}=(k+3) f_{n k}$ for $k \geqq n$, there is no $M>0$ such that $a_{n k} \leqq M \cdot f_{n k}$. Hence, this example also shows that the second hypothesis of Lemma 3.1.2 is not a necessary condition.

The results of $\$ 3.2$ hold for some quasi-Hausdorff-Nörlund methods. Ramanujan [10] has shown that for bounded $\left\{s_{n}\right\}$, if $\left\{s_{n}\right\}$ is summable Abel, then the $H^{*}\left(\mu_{n}\right)$ transform of $\left\{s_{n}\right\}$ is also summable Abel to the same sum. Also, it is easy to see that the alpha-matrix of $H^{*}\left(\mu_{n}\right)$ is $(C, 1)$-preserving, since

$$
\sum_{k=0}^{\infty} a_{n k}=\sum_{k=n}^{\infty}\left(\begin{array}{l}
k \\
n
\end{array}\right) \int_{0}^{1} u^{n}(1-u)^{k-n} d G=\int_{0}^{1}(1 / u) d G \text { for every } n .
$$

LEMмA 3.4.2. The alpha-matrix of $H^{*}\left(\mu_{n}\right)$ is monotone-preserving with respect to those nondecreasing sequences $\left\{p_{n}\right\}$ such that $q_{n}$ exists for every $n$ and

for every $n$.

$$
\lim _{k}\left(\begin{array}{l}
k+1 \\
n+1
\end{array}\right) \Delta^{k-n} \mu_{n} p_{k}=0
$$

Proof. For any $n \geqq 0$,

$$
\begin{aligned}
q_{n}-q_{n+1} & =\lim _{m}\left[\sum_{k=n}^{m+1}\left(\begin{array}{l}
k \\
n
\end{array}\right) \Delta^{k-n} \mu_{n} p_{k}-\sum_{k=n+1}^{m+1}\left(\begin{array}{c}
k \\
n+1
\end{array}\right) \Delta^{k-n-1} \mu_{n+1} p_{k}\right] \\
& =\lim _{m}\left[\sum_{1}-\sum_{2}\right] .
\end{aligned}
$$

In $\Sigma_{2}$ one can let $k=j+1$ and then replace $\Delta^{j-n} \mu_{n+1}$ by $\Delta^{j-n} \mu_{n}-\Delta^{j-n+1} \mu_{n}$. Then $\Sigma_{2}$ can be written as $\Sigma_{3}-\Sigma_{4}$. Letting $j+1=k$ in $\Sigma_{4}$, one finds that $\Sigma_{1}$ and $\Sigma_{4}$ can be combined. When the resulting expressions are simplified, one has 


$$
q_{n}-q_{n+1}=\lim _{m}\left[\sum_{k=n}^{m}\left(\begin{array}{c}
k+1 \\
n+1
\end{array}\right) \Delta^{k-n} \mu_{n}\left(p_{k}-p_{k+1}\right)+\left(\begin{array}{c}
m+2 \\
n+1
\end{array}\right) \Delta^{m+1-n} \mu_{n} p_{m+1}\right] .
$$

Hence,

$$
q_{n}-q_{n+1}=\sum_{k=n}^{\infty}\left(\begin{array}{l}
k+1 \\
n+1
\end{array}\right) \Delta^{k-n} \mu_{n}\left(p_{k}-p_{k+1}\right) \leqq 0
$$

since $\Delta^{p} \mu_{n} \geqq 0$.

Incidentally, the same kind of calculation, using $\mu_{n+1}$ in place of $\mu_{n}$, shows that the $T$-matrix of $H^{*}\left(\mu_{n}\right)$ preserves monotonicity of suitable sequences.

The Taylor method $T(a)$ of Fekete's original formulation is the quasi-Hausdorff method $H^{*}\left(a^{n}\right)$, where $0<a<1$. This method is generated by the function $G$, where $G(u)=0$ for $0 \leqq u \leqq a, G(u)=1$ for $a<u \leqq 1$. This observation, together with Lemma 3.4.1, shows that the alpha-matrix of any Taylor method transforms Nörlund sequences into Nörlund sequences. In fact, the alpha-matrix of $T(a)$ transforms nondecreasing Nörlund sequences into nondecreasing Nörlund sequences. To see this, let $\left\{p_{n}\right\}$ be such a sequence and let $p(x)=\Sigma p_{n} x^{n}$. Then $D^{n+1}[x \cdot p(x)] /(n+1) !=\sum_{k=n}^{\infty}\left(\begin{array}{l}k+1 \\ n+1\end{array}\right) p_{k} x^{k-n}$ converges for $|x|<1$, where $D=d / d x$. Then, for any $a, 0<a<1$, it follows that $\lim _{k} a^{n}\left(\begin{array}{c}k+1 \\ n+1\end{array}\right) p_{k}(1-a)^{k-n}=0$, and, by Lemma 3.4.2, the transform of $\left\{p_{n}\right\}$ by the alpha-matrix of $T(a)$ is nondecreasing.

\subsection{The Nörlund- $A$ method.}

\subsection{THE DEFINITION.}

Definition 4.1.1. Let $A$ be a regular summability method which has a $T$-matrix $\left(f_{n k}\right)$ with no zero rows and such that $f_{n k} \geqq 0$ for all $n$ and $k$. Let $\left(N, p_{n}\right)$ be a regular Nörlund method such that $Q_{n}=\sum f_{n k} P_{k}$ exists for each $n$, where $P_{n}=p_{0}+\cdots+p_{n}$. A sequence $\left\{s_{n}\right\}$ is said to be summable to $s$ by the Nörlund- $A$ method if and only if $T_{n} / Q_{n} \rightarrow s$, where $T_{n}=\sum f_{n k} S_{k}$ and $S_{n}=\sum_{k=0}^{n} p_{n-k} S_{k}$. Write $S_{n} \rightarrow s, G\left(A, p_{n}\right)$, in this case.

LEMMA 4.1.2. For any Nörlund sequence $\left\{p_{n}\right\}$ there exists an $r=r\left(p_{n}, A\right)>0$ such that for all $n, Q_{n} \geqq r$.

Proof. For all $n, P_{n} \geqq p_{0}$. Since $f_{n k} \geqq 0$, it follows from Theorem 9 in [4] that $\liminf Q_{n} \geqq \liminf P_{n} \geqq p_{0}$. Hence there exists an $N>0$ such that $Q_{n} \geqq p_{0} / 2$ for all $n \geqq N$. Furthermore, $Q_{n}>0$ for all $n$, since $\left(f_{n k}\right)$ has no zero rows. Set $r=\min \left(Q_{0}, Q_{1}, \cdots, Q_{N-1}, p_{0} / 2\right)$. Then for all $n, Q_{n} \geqq r>0$.

\subsection{Properties of $G\left(A, p_{n}\right)$.}

THEOREM 4.2.1. $G\left(A, p_{n}\right)$ is regular and includes $\left(N, p_{n}\right)$.

Proof. $\left.\quad T_{n} / Q_{n}=\left(\sum f_{n k} S_{k}\right) / \sum f_{n k} P_{k}\right)=\sum d_{n k}\left(S_{k} / P_{k}\right)$, where $d_{n k}=f_{n k} P_{k} / Q_{n}$. Then, $d_{n k} \leqq f_{n k} P_{k} / r \rightarrow 0$ as $n \rightarrow \infty$ for each $k$. Also, $\sum_{k}\left|d_{n k}\right|=\sum_{k} d_{n k}=1$ for every $n$. This shows that $\left(d_{n k}\right)$ is a $T$-matrix. Therefore, if $s_{n} \rightarrow s,\left(N, p_{n}\right)$, then $S_{k} / P_{k} \rightarrow s$. Hence, $T_{n} / Q_{n} \rightarrow s$. Since $\left(N, p_{n}\right)$ is regular, $G\left(A, p_{n}\right)$ is also regular. 
When $A$ is $(C, 1), p_{0}=p_{2}=1, p_{n}=0$ otherwise, and $s_{n}=(-1)^{n}$, it is easily seen that $s_{n} \rightarrow 0, G\left(A, p_{n}\right)$, but $\left\{s_{n}\right\}$ is not summable $\left(N, p_{n}\right)$. Thus, in general, $G\left(A, p_{n}\right)$ is not equivalent to $\left(N, p_{n}\right)$.

Definition 4.2.2 [6]. A summability method $A$ is said to be translative to the left if and only if $A-\lim r_{n}=A$-lim $s_{n}$ whenever $A$-lim $s_{n}$ exists and $r_{n}=s_{n-1}, r_{0}=0$.

THEOREM 4.2.3. If the method $A$ is translative to the left and if $\left\{p_{n}\right\}$ is a finite Nörlund sequence, then $G\left(A, p_{n}\right)$ includes $A$.

Proof. There exists an $N>0$ such that $P_{n}=P_{N}>0$ for all $n \geqq N$. Thus, $Q_{n} \rightarrow P_{N}$. Also, if $A$-lim $s_{n}=s$, then $T_{n}=p_{0} \cdot \sum_{k} f_{n k} s_{k}+p_{1} \cdot \sum_{k} f_{n k} s_{k-1}+\cdots+p_{N}$ $\cdot \sum_{k} f_{n k} s_{k-N} \rightarrow P_{N} s$. Therefore, $T_{n} / Q_{n} \rightarrow s$.

When $\left\{p_{n}\right\}$ has only finitely many nonzero terms and $A$ is translative to the left, $A$ is not, in general, equivalent to $G\left(A, p_{n}\right)$. For example, when $A$ is $(C, 1)$, $p_{0}=1, p_{1}=2, p_{2}=1$ and $p_{n}=0$ for $n \geqq 3$, the sequence $\left\{s_{n}\right\}$, with $s_{n}=(n+1)(-1)^{n}$, is not summable $A$, but $s_{n} \rightarrow 0,\left(N, p_{n}\right)$ and hence $s_{n} \rightarrow 0, G\left(A, p_{n}\right)$.

THEOREM 4.2.4. Let $\left(f_{n k}\right)$ and $\left(F_{n k}\right)$ be the T-matrices of the methods $A$ and $B$, both matrices having no zero rows and no negative elements. If there exists a matrix $\left(e_{n k}\right)$ with $e_{n k} \geqq 0$ for all $n$ and $k$, such that $\left(F_{n k}\right)=\left(e_{n k}\right)\left(f_{n k}\right)$ and $\lim _{n} e_{n k}=0$ for every $k$, then $G\left(B, p_{n}\right)$ totally includes $G\left(A, p_{n}\right)$ for all sequences $\left\{s_{n}\right\}$ for which the product $\left(e_{n k}\right)\left(f_{k j}\right)\left(S_{j}\right)$ is associative.

Proof. Let $T_{n}=\sum f_{n k} S_{k}, Q_{n}=\sum f_{n k} P_{k}, T_{n}^{*}=\sum F_{n k} S_{k}$ and $Q_{n}^{*}=\sum F_{n k} P_{k}$, all of which are assumed to exist for every $n$. Now, $T_{n}^{*}=\sum_{j} F_{n j} S_{j}=\Sigma_{k} e_{n k}\left(\sum_{j} f_{k j} S_{j}\right)$ $=\sum e_{n k} T_{k}$. Similarly, $Q_{n}^{*}=\sum e_{n k} Q_{k}$, the inversions being justified since all terms are non-negative. Then $T_{n}^{*} / Q_{n}^{*}=\sum_{k} d_{n k}\left(T_{k} / Q_{k}\right)$, where $d_{n k}=\left(e_{n k} Q_{k}\right) /\left(\sum_{k} e_{n k} Q_{k}\right)$. Now, $d_{n k} \leqq e_{n k} Q_{k} / r \rightarrow 0$ as $n \rightarrow \infty$ for each $k, r$ being that of Lemma 4.1.2 determined by $\left\{p_{n}\right\}$ and $B$. Also, $\sum_{k}\left|d_{n k}\right|=\sum_{k} d_{n k}=1$ for each $n$. Hence, $\left(d_{n k}\right)$ is a $T$-matrix with non-negative elements. Thus, if $T_{n} / Q_{n} \rightarrow s$, then $T_{n}^{*} / Q_{n}^{*} \rightarrow S$ for $s$ finite or infinite, and the assertion is proved.

Clearly, if $\left\{s_{n}\right\}$ is a sequence for which both $\sum f_{n k}\left|S_{k}\right|$ and $\sum F_{n k}\left|S_{k}\right|$ converge, then the product $\left(e_{n k}\right)\left(f_{k j}\right)\left(S_{j}\right)$ is associative. Also, if $\left\{s_{n}\right\}$ is such that $\left\{S_{n}\right\}$ lies in an angle less than $\pi$, in the sense of [1, p. 124], then the proof of 5.7 III on p. 124 of [1] shows that this product again is associative. Another simple case of associativity arises when both matrices $\left(e_{n k}\right)$ and $\left(f_{n k}\right)$ are row-finite. In the latter case, $G\left(B, p_{n}\right)$ totally includes $G\left(A, p_{n}\right)$ for all sequences $\left\{s_{n}\right\}$.

If the matrices $\left(f_{n k}\right)$ and $\left(F_{n k}\right)$ are lower-triangular, that is, if they have only zeros above the main diagonal, and if $f_{n n} \neq 0$ for all $n$, then there exists a twosided reciprocal of $\left(f_{n k}\right)$ which is a lower-triangular matrix [1, pp. 19-22]. If $\left(f_{n k}\right)^{-1}$ denotes this reciprocal, the product matrix $\left(F_{n k}\right)\left(f_{n k}\right)^{-1}$ suffices for $\left(e_{n k}\right)$ in the above theorem, provided that this product matrix has all of its elements non-negative and has column-limits zero. 
Similarly, if $\left(f_{n k}\right)$ and $\left(F_{n k}\right)$ are upper-triangular, that is, if they have only zeros below the main diagonal, and if $f_{n n} \neq 0$ for all $n$, then there exists a left-hand reciprocal of $\left(f_{n k}\right)$ which is also an upper-triangular matrix $[1, \mathrm{pp} .6,19]$. The product matrix $\left(F_{n k}\right)\left(f_{n k}\right)_{L}^{-1}$, where $\left(f_{n k}\right)_{L}^{-1}$ denotes this reciprocal, exists and would suffice for $\left(e_{n k}\right)$ of Theorem 4.2.4 provided that this product matrix has all its elements non-negative.

4.3. NörLuND-HAuSDORFF METHODS. The $H\left(\mu_{n}\right)$ methods, defined in $\S 3.3$ above, satisfy the hypotheses for $A$ of Definition 4.1.1. The following considerations show how the results of $\S 4.2$ hold for $G\left(H\left(\mu_{n}\right), p_{n}\right)$.

The $(C, k)$ and Euler methods, which are $H\left(\mu_{n}\right)$ methods, are translative to the left [2, p. 419], [14, p. 131]. Therefore, $G\left(H\left(\mu_{n}\right), p_{n}\right)$ includes $H\left(\mu_{n}\right)$ for finite Nörlund sequences in these cases. The same result holds for all $H\left(\mu_{n}\right)$ methods if only bounded sequences are considered, for Parameswaran [7] has shown that any regular Hausdorff method is translative to the left for such sequences.

The $T$-matrix $\left(f_{n k}\right)$ of $H\left(\mu_{n}\right)$ has a two-sided reciprocal of the form $\left(c_{n k}\right)$, where $c_{n k}=\left(\begin{array}{l}n \\ k\end{array}\right) \Delta^{n-k}\left(1 / \mu_{k}\right)$ when $n \geqq k, 0$ otherwise [4, p. 262]. If $\left(F_{i k k}\right)$ is the $T$ matrix of another method, $H\left(\mu_{n}^{*}\right)$, then $\left(F_{n k}\right)\left(c_{n k}\right)$ exists and equals $\left(e_{n k}\right)$, where $e_{n k}=\left(\begin{array}{l}n \\ k\end{array}\right) \Delta^{n-k}\left(\mu_{k}^{*} / \mu_{k}\right) \quad$ when $n \geqq k, 0$ otherwise. The hypotheses of Theorem 4.2.4 would be satisfied when $\Delta^{p}\left(\mu_{n}^{*} / \mu_{n}\right) \geqq 0$ for $n \geqq 0, p \geqq 0$ and $\lim _{n} \Delta^{n}\left(\mu_{0}^{*} / \mu_{0}\right)=0$ [4, pp. 252-255].

4.4. NÖRLUND-QUASI-HAUSDORFF METHODS. If $\mu_{n} \neq 0$ for all $n$, the $H^{*}\left(\mu_{n}\right)$ methods of $\$ 3.4$ satisfy the hypotheses for $A$ in Definition 4.1.1. The results of $\$ 4.2$ hold for some of these methods.

Any regular quasi-Hausdorff method is translative to the left for bounded sequences [11]. Therefore, when $\mu_{n} \neq 0$ for all $n, G\left(H^{*}\left(\mu_{n}\right), p_{n}\right)$ includes $H^{*}\left(\mu_{n}\right)$ for finite Nörlund sequences $\left\{p_{n}\right\}$ and bounded $\left\{s_{n}\right\}$.

The $T$-matrix $\left(f_{n k}\right)$ of $H^{*}\left(\mu_{n}\right)$, when $\mu_{n} \neq 0$ for all $n$, has a two-sided reciprocal of the form $\left(d_{n k}\right)$, where $d_{n k}=\left(\begin{array}{l}k \\ n\end{array}\right) \Delta^{k-n}\left(1 / \mu_{n+1}\right)$ when $k \geqq n, 0$ otherwise. If $\left(F_{n k}\right)$ is the $T$-matrix of another method $H^{*}\left(\mu_{n}^{*}\right)$, then $\left(F_{n k}\right)\left(d_{n k}\right)$ exists and equals $\left(e_{n k}\right)$, where $e_{n k}=\left(\begin{array}{l}k \\ n\end{array}\right) \Delta^{k-n}\left(\mu_{n+1}^{*} / \mu_{n+1}\right)$ when $k \geqq n, 0$ otherwise. Thus, the matrices of these methods would satisfy the hypotheses of Theorem 4.2.4 whenever $\Delta^{p}\left(\mu_{n+1}^{*} / \mu_{n+1}\right) \geqq 0$ for all $n$ and $p$.

5.0. Comparison of the methods $F\left(A, p_{n}\right)$ and $G\left(A, p_{n}\right)$. The methods $F\left(A, p_{n}\right)$ and $G\left(A, p_{n}\right)$, in general, are not comparable, for let $A$ be the method which transforms the sequence $\left\{s_{n}\right\}$ into the sequence $\left\{t_{n}\right\}$, where $t_{n}=\left(2 s_{n}+s_{n+1}\right) / 3$. A short calculation shows that the alpha-matrix of $A$ is $\left(a_{n k}\right)$, where $a_{00}=1$, $a_{01}=1 / 3, a_{n n}=2 / 3$ and $a_{n, n+1}=1 / 3$ for $n \geqq 1, a_{n k}=0$ otherwise. The matrices for $A$ satisfy Lemma 3.1.2 with $M=2$, and also satisfy Definition 4.1.1. Let $\left(N, p_{n}\right)$ be defined by $p_{0}=1, p_{1}=3, p_{n}=0$ for $n \geqq 2$. Then $q_{0}=q_{1}=2, q_{n}=0$ for $n \geqq 2$. If $s_{n}=3(-1)^{n+1}$, one can easily see that $t_{n}=(-1)^{n+1}$ and that $t_{n} \rightarrow 0,\left(N, q_{n}\right)$. However, $T_{n} / Q_{n}=(-1)^{n} / 2$ and $\left\{s_{n}\right\}$ is not summable $G\left(A, p_{n}\right)$. 
On the other hand, if $s_{n}^{*}=(-3)^{n+1}$, then $T_{n}^{*}=0$ for $n \geqq 1$, so $s_{n}^{*} \rightarrow 0, G\left(A, p_{n}\right)$, while $\left(q_{0} t_{n}^{*}+q_{1} t_{n-1}^{*}\right) / Q_{n}=-(-3)^{n-1}$ and $\left\{s_{n}^{*}\right\}$ is not summable $F\left(A, p_{n}\right)$.

However, the two methods coincide when $A$ is the Euler method $(E, r)$, defined as the Hausdorff method $H\left(\mu_{n}\right)$, where $\mu_{n}=(1+r)^{-n}$ for positive $r[4, \mathrm{p} .248]$.

THEOREM 5.0.1. $F\left((E, r), p_{n}\right) \equiv G\left((E, r), p_{n}\right)$.

Proof. It suffices to show that $\sum q_{n-k} t_{k} \equiv T_{n}$ for all $n$. Now, $q_{0}=p_{0}$ and, for $n \geqq 1$,

Also,

$$
q_{n}=(1+r)^{-n} \cdot \sum_{j=1}^{n}\left(\begin{array}{c}
n-1 \\
j-1
\end{array}\right) r^{n-j} p_{j}
$$

$$
t_{n}=(1+r)^{-n} \cdot \sum_{m=0}^{n}\left(\begin{array}{c}
n \\
m
\end{array}\right) r^{n-m} s_{m} \text { for all } n .
$$

After combining terms, letting $i=m+j$ and simplifying, one sees that

$$
\begin{aligned}
\sum_{k=0}^{n} q_{n-k} t_{k} & =(1+r)^{-n} \cdot \sum_{j=0}^{n} \sum_{i=j}^{n}\left(\begin{array}{l}
n \\
i
\end{array}\right) r^{n-i} p_{j} s_{i-j} \\
& =(1+r)^{-n} \cdot \sum_{i=0}^{n}\left(\begin{array}{c}
n \\
i
\end{array}\right) r^{n-i} S_{i}=T_{n} .
\end{aligned}
$$

A related result for Taylor-Nörlund and Nörlund-Taylor methods is the following theorem.

THEOREM 5.0.2. For sequences $\left\{s_{n}\right\}$ which have $T(a)$-transforms, $F\left(T(a), p_{n}\right)$ $\equiv G\left(T(a), p_{n}\right)$.

Proof. As in the previous theorem, it suffices to show that $\sum q_{n-k} t_{k} \equiv T_{n}$ for all $n$, assuming that $t_{n}$ exists for each $n$. Now, the series defining $q_{j}$ converge absolutely for each $j$, and hence, by Mertens' theorem [5, p. 321], $q_{j} t_{n-j}=\Sigma_{k} c_{k}^{(j)}$, the Cauchy product of these series. Then,

Now,

$$
\sum_{j=0}^{n} q_{j} t_{n-j}=\sum_{j=0}^{n} \sum_{k=0}^{\infty} c_{k}^{(j)}=\sum_{k=0}^{\infty} \sum_{j=0}^{n} c_{k}^{(j)}
$$

$$
\sum_{j=0}^{n} c_{k}^{(j)}=a^{n+1}(1-a)^{k} \cdot \sum_{j=0}^{n} \sum_{r=0}^{k}\left(\begin{array}{c}
r+j \\
j
\end{array}\right)\left(\begin{array}{c}
k+n-j-r \\
n-j
\end{array}\right) p_{r+j} s_{k+n-j-r}
$$

When the rectangular array $(j, r)$ is summed along the diagonals $j+r=i$, $i=0, \cdots, n+k$, and the resulting expressions are simplified and combined, one sees that

$$
\sum_{j=0}^{n} q_{j} t_{n-j}=\sum_{k=0}^{\infty}\left(\begin{array}{c}
n+k \\
n
\end{array}\right) a^{n+1}(1-a)^{k} S_{k+n}=T_{n} .
$$

It follows immediately from this theorem that $G\left(T(a), p_{n}\right)$ includes $F\left(T(A), p_{n}\right)$. However, when $s_{n}=(a-1)^{-n},\left\{s_{n}\right\}$ fails to have a $T(a)$-transform, so $\left\{s_{n}\right\}$ is not 
summable $F\left(T(a), P_{n}\right)$ for any Nörlund sequence, although $s_{n} \rightarrow 0, G\left(T(a), p_{n}\right)$ when $p_{0}=1, p_{1}=(1-a)^{-1}, p_{n}=0$ for $n \geqq 2$.

6.0. Series-to-sequence analogues. The methods $F\left(A, p_{n}\right)$ and $G\left(A, p_{n}\right)$, which were developed above, are applicable to the sequence of partial sums of a series. In this section, analogous methods are defined which operate directly on the terms of the series.

\subsection{THE $A$-NÖRLUND METHOD.}

Definition 6.1.1. Let $A$ be a regular summability method with alpha-matrix $\left(a_{n k}\right)$ and gamma-matrix $\left(g_{n k}\right)$. Let $\left(N, p_{n}\right)$ be a regular Nörlund method such that $\left(N, q_{n}\right)$ is a regular Nörlund method, where $q_{n}=\sum a_{n k} p_{k}$. A series $\sum u_{k}$ is said to be summable to $s$ by the $A$-Nörlund method if and only if $t_{n}^{\prime} \rightarrow s,\left(N, q_{n}\right)$, where $t_{n}^{\prime}=\sum g_{n k} u_{k}$. Write $\sum u_{k}=s, F^{\prime}\left(A, p_{n}\right)$ in this case.

If $\left(a_{n k}\right)$ is the alpha-matrix of $A$ and $v_{n}=\sum a_{n k} u_{k}$, Vermes [13] has shown that $v_{0}+\cdots+v_{n}=t_{n}^{\prime}$. Therefore, an equivalent form of Definition 6.1.1 is: $\Sigma u_{k}=s$, $F^{\prime}\left(A, p_{n}\right)$, if and only if $\Sigma v_{n}=s,\left(N, q_{n}\right)$.

Definition 6.1.1 is more general than Definition 3.1.1, since there exist regular summability methods to which correspond alpha- and gamma-matrices, but not $T$-matrices [1, p. 87]. However, if a method has a $T$-matrix, then there always exist alpha- and gamma-matrices for that method. The explicit form of these matrices is given in $\$ 2.0$ above. The relation between $F\left(A, p_{n}\right)$ and $F^{\prime}\left(A, p_{n}\right)$, when $A$ has a $T$-matrix, is investigated in the next theorem.

THEOREM 6.1.2. Let $A$ have a T-matrix $\left(f_{n k}\right)$ and a gamma-matrix $\left(g_{n k}\right)$. Let $s_{n}=u_{0}+\cdots+u_{n}$ be such that $\sum f_{n k} s_{k}, \sum g_{n k} u_{k}$ and $\lim _{k} g_{n k} s_{k}=w_{n}$ exist for each $n$. If $w_{n} \rightarrow 0,\left(N, q_{n}\right)$, then $s_{n} \rightarrow s, F\left(A, p_{n}\right)$, if and only if $\sum u_{k}=s, F^{\prime}\left(A, p_{n}\right)$.

Proof. Let $s_{-1}=0$. As on p. 86 of [1],

$$
\begin{aligned}
\sum_{k=0}^{\infty} f_{n k} s_{k} & =\lim _{m}\left[\sum_{k=0}^{m} g_{n k} s_{k}-\sum_{k=0}^{m} g_{n, k+1} s_{k}\right] \\
& =\lim _{m}\left[\sum_{k=0}^{m+1} g_{n k}\left(s_{k}-s_{k-1}\right)-g_{n, m+1} s_{m+1}\right] .
\end{aligned}
$$

This shows that, in the notation of 3.1.1 and 6.1.1 above, $t_{n}+w_{n}=t_{n}^{\prime}$ for every $n$, and the assertion of the theorem follows.

If $\left\{s_{n}\right\}$ is bounded, then $\lim _{k} g_{n k} s_{k}=0$ for every $n$, since $\lim _{k} g_{n k}=0$ when $A$ has a $T$-matrix $\left[1\right.$, p. 86]. Hence, for series with bounded partial sums, $t_{n}=t_{n}^{\prime}$, and the methods $F\left(A, p_{n}\right)$ and $F^{\prime}\left(A, p_{n}\right)$ are identical. Another case in which these methods are identical is when the gamma-matrix of $A$ has only finitely many nonzero terms in each row, for then, $\lim _{k} g_{n k} s_{k}=0$ for every sequence $\left\{s_{n}\right\}$.

6.2. THE NÖRLUND- $A$ METHOD. A natural series-to-sequence analogue of Definition 4.1.1 would use the series-to-sequence form for $\left(N, p_{n}\right)$ as follows: A series $\sum u_{k}$ is summable to $s$ by the Nörlund- $A$ method if and only if $T_{n} / Q_{n} \rightarrow^{-}$, 
where $T_{n}=\Sigma f_{n k} S_{k}, S_{n}=P_{n} u_{0}+\cdots+P_{0} u_{n}, P_{n}=p_{0}+\cdots+p_{n}$ and $Q_{n}=\Sigma f_{n k} P_{k}$. However, since the expressions $P_{n} u_{0}+\cdots+P_{0} u_{n}$ and $p_{n} s_{0}+\cdots+p_{0} s_{n}$ are identical, this series-to-sequence analogue of $G\left(A, p_{n}\right)$ is identical to applying $G\left(A, p_{n}\right)$ to the sequence of partial sums of $\sum u_{k}$.

6.3. The GeOMETRIC SERIES. The regions of the complex plane in which the geometric series is summable $(E, r)$ and $T(a)$ are well known [4, p. 178], [13]. For both methods, a series-to-series transformation changes the geometric series into a series of the form $\sum b_{n} X^{n}$, where $X=X(z, r)$ or $X=X(z, a)$. The regions of summability result from imposing the conditions that $X$ exist and $|X|<1$. These regions can be extended by the methods defined above.

THEOREM 6.3.1. If $\left\{p_{n}\right\}$ is a nondecreasing Nörlund sequence with $p_{0}(r+1) \leqq p_{1}$, then $\sum z^{k}=1 /(1-z), F^{\prime}\left((E, r), p_{n}\right)$ for all $z$ satisfying $|r+z| \leqq r+1, z \neq+1$.

Proof. By Lemma 3.3.1 and Theorem 3.2.6, it suffices to consider the case $\left(N, q_{n}\right)=(C, 1)$. Upon transforming the geometric series by the alpha-matrix of $(E, r)$, one finds that $v_{0}=1$, and, when $n \geqq 1$,

$$
v_{n}=(1+r)^{-n} \cdot \sum_{j=1}^{n}\left(\begin{array}{c}
n-1 \\
j-1
\end{array}\right) r^{n-j_{z} j}=\frac{c_{n} z}{1+r},
$$

where $c_{n}=[(r+z) /(1+r)]^{n-1}$. Now, for all $z$ such that $|r+z| \leqq r+1, z \neq+1$, $\sum_{k=1}^{\infty} c_{k}=[1-(r+z) /(r+1)]^{-1},(C, 1)[5$, p. 481]. By Theorem 47 of [4], it follows that $(C, 1)-\sum v_{k}=1+[z /(1+r)] \cdot(C, 1)-\sum c_{k}=1 /(1-z)$ for these $z$ 's.

THEOREM 6.3.2. If $\left\{p_{n}\right\}$ is a nondecreasing Nörlund sequence, then $\sum z^{k}$ $=1 /(1-z), F^{\prime}\left(T(a), p_{n}\right)$ for all $z$ such that $z \neq+1,|z|<1 /(1-a),|a z|$ $\leqq|1-(1-a) z|$.

Proof. It follows from the discussion at the end of $\$ 3.4$ above that, as in the previous theorem, it suffices to consider the case $\left(N, q_{n}\right)=(C, 1)$. Using the alpha-matrix for $T(a)$, one gets for all $n, v_{n}=a^{n} \cdot \sum_{k=n}^{\infty}\left(\begin{array}{l}k \\ n\end{array}\right)(1-a)^{k-n} z^{k}$ $=(a z)^{n} /[1-(1-a) z]^{n+1}$ if and only if $(1-a)|z|<1$. Then, $\Sigma v_{k}$ $=[1-(1-a) z]^{-1} \cdot \Sigma[a z /(1-z+a z)]^{k}=1 /(1-z),(C, 1)$, if and only if $|a z| \leqq|1-(1-a) z|, z \neq+1[5$, p. 481].

If one transforms $s_{n}=1+z+\cdots+z^{n}$ by the $T$-matrix of $T(a)$, one finds that $t_{n}=1 /(1-z)-a z \cdot v_{n} /(1-z)$ if and only if $(1-a)|z|<1$. Since $\sum v_{n}$ is $(C, 1)$-summable whenever $z \neq+1,|a z| \leqq|1-(1-a) z|$, then $v_{n} \rightarrow 0,(C, 1)$ $\left[5\right.$, p. 485]. Hence for these $z$ 's, $t_{n} \rightarrow 1 /(1-z),(C, 1)$, so the assertion of Theorem 6.3.2 holds for $F\left(T(a), p_{n}\right)$ and $G\left(T(a), p_{n}\right)$ as well.

NoTE. The material in this paper stems from the author's recent doctoral dissertation at the University of Pittsburgh. The author wishes to acknowledge the guidance of his advisor, Professor G. Laush, who suggested the problem. $\mathrm{He}$ also wishes to thank the referee for his helpful comments and for his suggestions about the addition of material on total inclusion. 


\section{BIBLIOGRAPHY}

1. R. G. Cooke, Infinite matrices and sequence spaces, Macmillan, London, 1950.

2. P. Dienes, The Taylor series, Dover, New York, 1957.

3. M. Fekete, New methods of summability, J. London Math. Soc. 33 (1958), 466-470.

4. G. H. Hardy, Divergent series, Oxford Univ. Press, Oxford, 1949.

5. K. Knopp, Theory and application of infinite series, 2nd English ed., Translated by R. C. Young, Hafner, New York, 1951.

6. B. Kuttner, The problem of 'translativity' for Hausdorff summability, Proc. London Math. Soc. 6 (1956), 117-138.

7. M. R. Parameswaran, On the translativity of Hausdorff- and some related methods of summability, J. Indian Math. Soc. (N.S.) 23 (1959), 45-64.

8. T. Pati, Products of summability methods, Proc. Nat. Inst. Sci. India Part A 20 (1954), 348-351.

9. M. S. Ramanujan, On Hausdorff and quasi-Hausdorff methods of summability, Quart. J. Math. Oxford Ser (2) 8 (1957), 197-213.

10. - Theorems on the product of quasi-Hausdorff and Abel transforms, Math. Z. 64 (1956), 442-447.

11. — The 'translativity' problem for quasi-Hausdorff methods of summability, Proc. Nat. Inst. Sci. India Part A 24 (1958), 4-14.

12. B. E. Rhoades, Hausdorff summability methods, Trans. Amer. Math. Soc. 101 (1961), 396-425.

13. P. Vermes, Series to series transformations and analytic continuation by matrix methods, Amer. J. Math. 71 (1949), 541-562.

14. K. Zeller, Theorie der Limitierungsverfahren, Ergebnisse der Mathematik und Ihrer Grenzgebiete (N.S.), no. 15, Springer, Berlin, 1958.

\section{STATE University OF New York.}

Albany, New York 